\title{
Construction of Machining and Simulation System for Ball-end Milling
}

\author{
Shuai Li, Hong Min
}

College of Mechanical and Electrical Engineering, Zaozhuang University Shandong province, 277160

\begin{abstract}
With the development of science and technology, more and more complex surfaces are used in mechanical products. The application and processing of complex surface have become an important part of mechanical research. The ball end milling cutter is widely used as the main tool for machining complex surfaces. In this paper, in order to shorten the development cycle and avoid the waste of time and materials brought by on-site test, a virtual simulation system for ball end milling cutter is established on the Solidworks platform, which verifies the rationality and correctness of the previous modeling, and provides a theoretical basis for the optimization of the grinding parameters. This method avoids the difficulties in the research, reduces the workload and has certain reference value and practical significance for the designer.
\end{abstract}

Keywords: Ball nose end mill; Solidworks; Secondary development; Parametric design; simulation

\section{球头立铣刀加工仿真系统的建立}

\author{
李帅闵红 \\ (疋庄学院 机电工程学院, 山东 本庄 $277160 ;$ )
}

摘要: 随着科学技术的发展, 机械产品中采用了越来越多的复杂曲面, 复杂曲面的应用和加工已成为机械学科研究的重 要内容。而球头立铣刀作为复杂曲面加工的主要刀具, 其应用越来越广泛。为了缩短研发周期, 避免现场试切带来的时间和 材料上的浪费，我们在 Solidworks 平台上建立了球头立铣刀虚拟仿真加工系统，验证前期建模的合理性和正确性，为后期 刃磨参数的优化提供了理论依据。该方法避开了研究中的难点, 减少了工作量, 对于设计人员具有一定的参考价值和现实意 义。

关键词: 球头立铣刀; Solidworks; 二次开发；参数化设计；仿真

中图分类号: TP278 文献标志码: A

\section{引言}

随着制造技术的不断发展，零件的外形越来越复杂。各种先进的数控加工设备为复杂曲面的加工提供 了硬件基础, 各种 $\mathrm{CAD}$ 应用软件为复杂曲面的设计提供了先进的手段, 各种 CAM 软件充分发挥了数控机床 高精度和高效率的制造特点 [1]。复杂曲面加工手段的发展又促进了复杂曲面应用的研究和发展。

就仿真加工而言, 按仿真对象考察方式的不同一般可分为纯几何仿真 (Puerly Geometric Simulation) 和物理仿真 (Physical Simulation)。前者不考虑切削参数、切削力及其他物理因素的影响, 只仿真刀 具一工件几何体的运动 [2]。后者运用物理规律去模拟被仿真的事件, 此时要考虑力、速度、质量、密度、 能量以及其他物理参数的影响, 因此这种方法比较复杂, 而且随着应用对象和访问目的的不同而有很大的 变化, 现在市场上的 CAM 软件大部分只提供几何仿真功能 [3]。对于物理仿真, 由于涉及对刀具切削过程 物理规律的研究，大部分软件没有提供相应的功能，因此我们所用的仿真方法是纯几何仿真。

SolidWorks 是一套完全基于 Windows 平台的全参数化特征造型软件，以其强大的功能、友好的界面 和不断创新的技术而逐渐成为主流的三维终端 CAD 解决方案。SolidWorks 通过对象链接与嵌入 (即 OLE 技 
术) 为用户提供了大量的 SolidWorks API 函数，凡支持 OLE 编程的开发工具均可用于 SolidWorks 的二次 开发, 创建出用户定制的、专用的 SolidWorks 功能模块。SolidWorks 还可与 Cosmos 等有限元分析软件无 缝连接, 实现仿真分析 $[4]$ 。

但是 SolidWorks 也并不可能完全满足特定企业的特殊要求。例如没有适合我国国标的标准件库等等。 因此, 为了适应企业的特殊需要, 使 SolidWorks 在我国企业中有效发挥作用, 并使常用或重复的任务简 单化，提高工作效率，就必须对其进行本地化和专业化的二次开发。

\section{1 直接实体造型法的原理}

直接实体造型法是指对工件体与刀具运动所形成的包络体进行实体布尔差运算，工件体的三维模型随 着切削过程被不断更新的造型方法[5]。采用 CSG (Constructive Solid Geometry) 法来记录毛坏的三维模 型, 利用一些基本图元如长方体、圆柱体、圆雉体等, 和集合运算, 特别是并运算, 将毛坏和一系列刀具 扫描过的区域记录下来，然后应用集合差运算从毛坏中顺序除去扫描过的区域。逐步的切削仿真利用毛坏 和切削区域的差运算来实现, 毛坏的显示采用了深度缓冲区算法, 将毛坏划分为多边形实现毛坏的可视化 $[6]$ 。

\section{2 仿真系统平台及二次开发工具的选取}

通过综合考虑各种三维设计软件平台及二次开发的功能特性等特点, 本文选取 Solidworks 软件进行 开发, 这取决于 Solidworks 的先进性、可行性。SolidWorks 是Windows 原创的三维实体设计软件, 全面 支持微软的 OLE 技术。它通过 OLE 对象链接与嵌入 (Object Linking and. Embedding) 技术为用户提供 了大量的 SolidWorks API 函数[7]。SolidWorks 的 API (Application Programming Interface) 应用编 程接口, 是一个基于 oLE Automation 的编程接口, 其中包含了数以百计的功能函数, 函数对象结构图如 图 3-1 所示。这些函数提供了程序员直接访问 SolidWorks 的能力, 可以被 VB、 C / C ++等编程语言调用, 从

SolidWorks 的二次开发工具很多, 例如 C 语言、C++语言、Visual C++、Visual Basic、VBA 等。开 发者可以根据自身的条件、工具的特点选择一种合适的开发工具。当然也可以同时选用两三种工具, 但这 对开发者的要求极高 [8]。VB 是目前Windows 平台下的主流开发工具之一, 支持各类最新的Windows 技术, VB 对 OLE 的支持有多种方式, 如使用 OLE 控件, 或在运行时创建 OLE 对象等方法。通常, 为了较好地对对 象进行控制, 多采用程序运行时再创建 OLE 对象的方法 [9]。同时, 由于 Visual Basic 具有不可取代的简 单易用性和真正的 “所见即所得” 特性, 使用Visual Basic 来开发软件, 开发周期短, 代码效率高。 


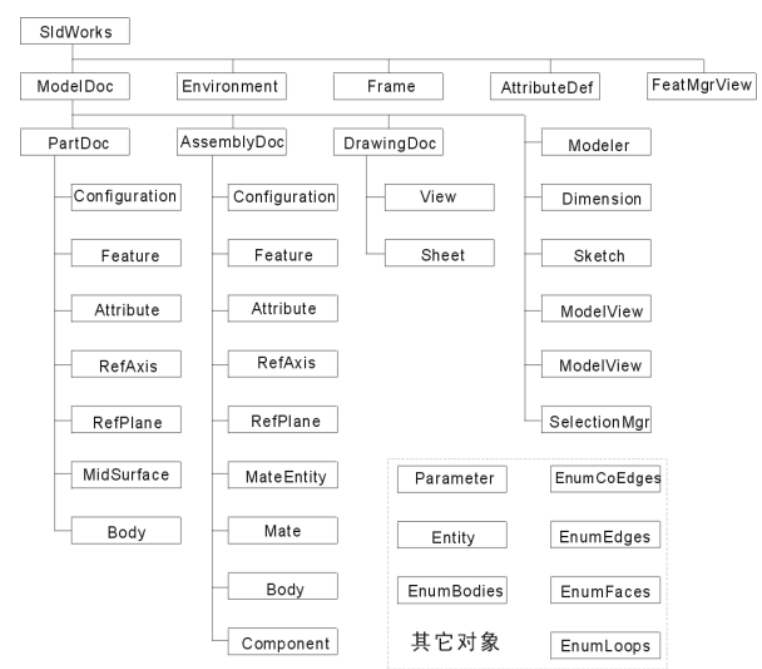

图 1 SolidWorks API 对象结构图

\section{3 球头立铣刀和砂轮的参数化建模}

在球头立铣刀的刃磨仿真加工研究中, 为了优化刃磨参数或进行误差分析, 实验需要建立大量不同尺 寸、不同类型的铣刀模型和砂轮模型, 为了避免重复性劳动, 缩短研发周期, 必须对其进行参数化设计。 参数化设计方法就是将模型中的定量信息变量化, 使之成为任意调整的参数。对于变量化参数赋予不同数 值, 就可得到不同大小和形状的零件模型。

为了避免生成过多的㝋余代码, 使驱动参数尺寸尽可能的少, 尽可能简化特征建立的步骤。首先打开 宏录制, 在 SolidWorks 中创建实体并用变量标注参数尺寸, 如图 2 所示。

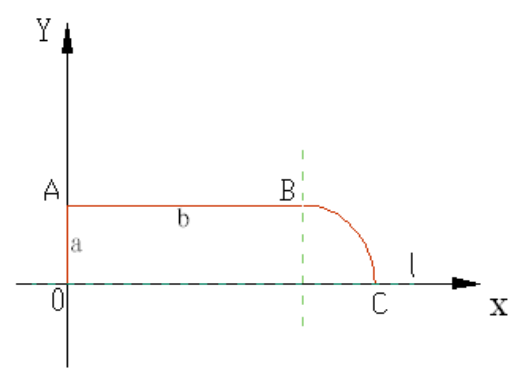

图 2 铣刀毛坏草图

在 VB 中设计人机交互式界面，窗体上有两个 TextBox 控件用于控制铣刀半径和刀柄长度，设置时将 两个文本框的变量与草图中的 $\mathrm{a}, \mathrm{b}$ 变量关联起来。两个 CommandButton 控件分别用于执行实体的生成和 退出, 设计好的用户界面见图 3。

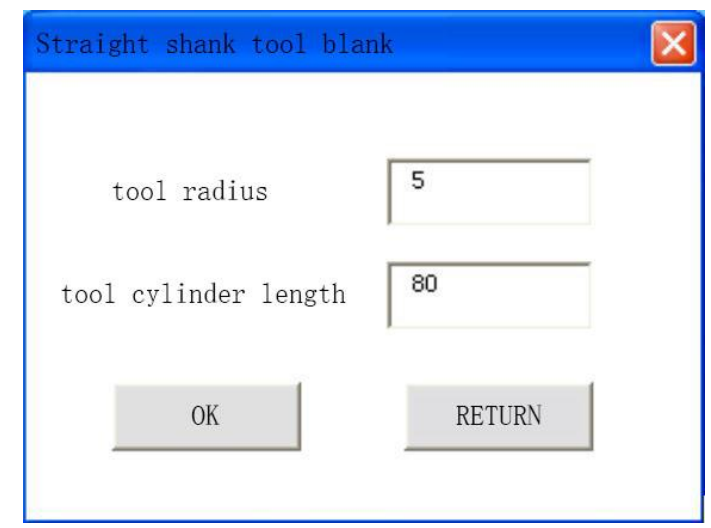

图 3 人机交互界面 
由于前面用宏录制得到了实体建立的基础代码，根据宏文件中的对象和方法在自己的应用程序中合理 组织, 稍做修改, 对具体参数用变量代替, 这样通过对变量的控制可实现球头立铣刀毛坏的参数化建模。 在二次开发过程中，宏录制文件中的许多对象、方法可以直接在 Visual Basic 应用程序中应用，这就大大 简化了开发过程。程序代码编写好以后, 生成 exe 可执行文件, 也可以做为一个插件添加到 SolidWorks 的 界面中去。设计者只要输入半径和刀具圆柱长度参数, 即可自动生成实体模型, 大大提高了工作效率。这 样就完成了球头立铣刀毛坏的参数化建模。执行上述程序，运行结果如图 4。

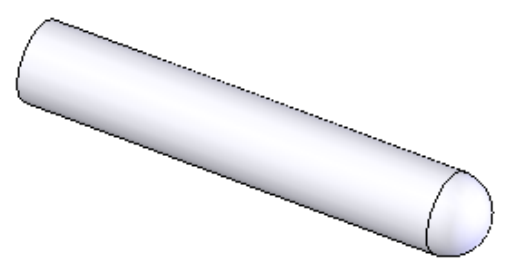

图 4 铣刀毛坏

根据上述的方法, 对砂轮进行参数化建模, 其用户界面及运行结果如图 5、图 6 所示。

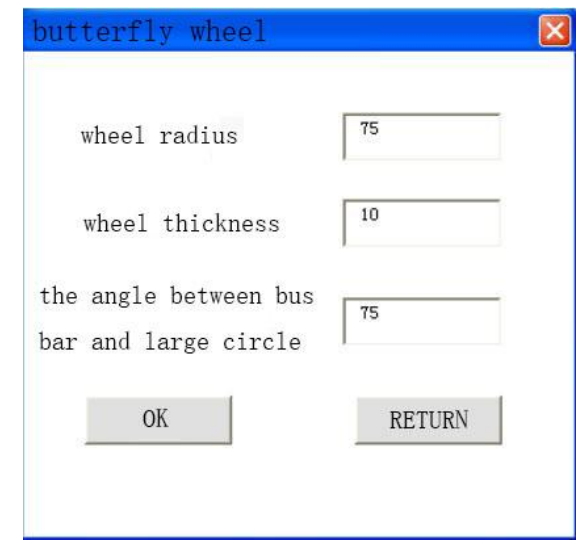

图 5 碟形砂轮界面

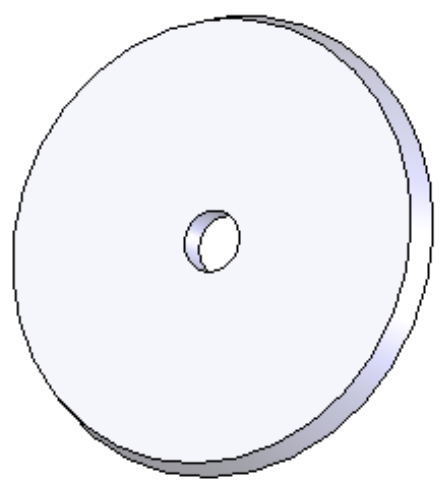

图 6 碟形砂轮

\section{4 仿真加工系统的建立}

依据实际加工过程, 以 SolidWorks 为操作平台, VB 为开发工具, 建立球头立铣刀仿真加工系统。此 加工虚拟系统充分利用 SolidWorks API 的方法、属性, 选用直接实体造型法来实现球头立铣刀前刀面的刀 磨仿真[10]。按照球头立铣刀前刀面形成的位置关系编制合理的运动程序, 调用铣刀毛坏模型和砂轮模型, 用程序控制砂轮的移动和偏转, 通过实体间的布尔差运算 (即 SolidWorks 菜单里的组合操作), 实现砂轮 实体对铣刀毛坏实体的切除加工, 从而形成球刀前刀面和周刃前刀面, 如图 7 所示。

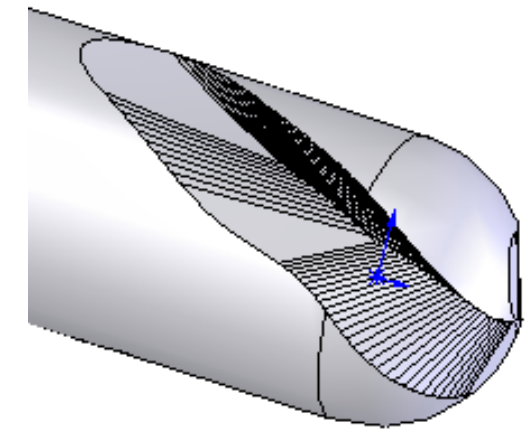

(a)

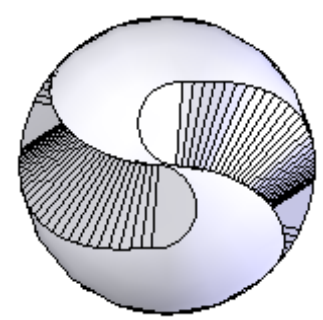

(b)

图 7 前刀面刃磨仿真结果 


\section{5 仿真结果的分析及评价}

球头立铣刀周刀前刀面与球刀前刀面的光滑连接对球头立铣刀的切削性能、加工零件的表面质量以及 球头立铣刀的排屑性能等都有非常重要的影响, 球头立铣刀周刀前刀面与球刀前刀面的光滑连接问题已引 起广泛重视[11]。本研究在球刀前刀面磨削加工过程中, 采取砂轮的大圆始终与球刀前刀面螺旋槽槽底相 切的方法确定砂轮与工件的相对运动关系, 圆柱部分圆周螺旋槽始终采取与普通螺旋槽磨削加工的方式加 工, 从仿真结果可以看出（图 8), 较好的实现了周刃前刀面与球刃前刀面的光滑连接。
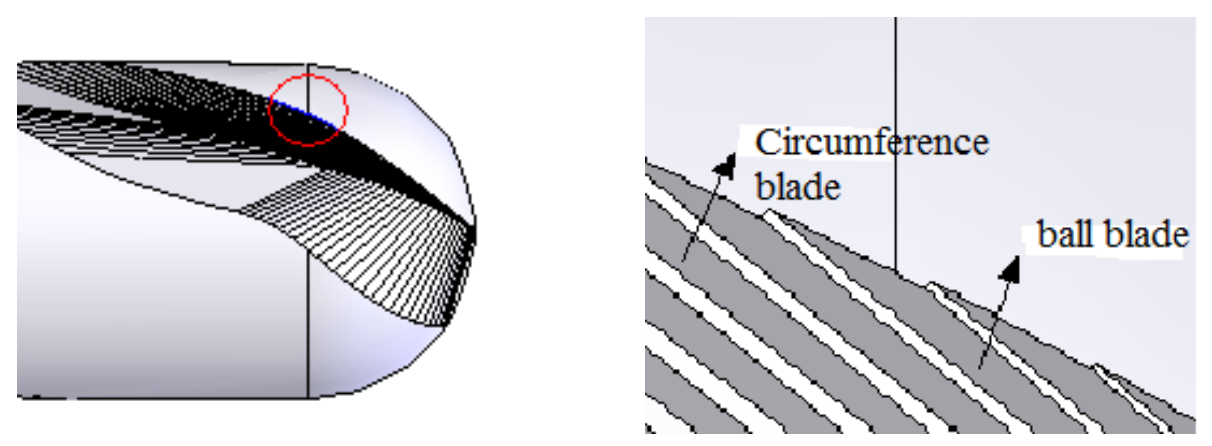

图 8 球刃和周刃的光滑连接

球头立铣刀刃磨仿真发现, 在靠近刃口部分有一个很窄的刃带（图 9)。该刃带为正倒棱, 能起到卷 屑、排屑的作用, 有利于切削液的渗透和增加刀具刃口的强度。

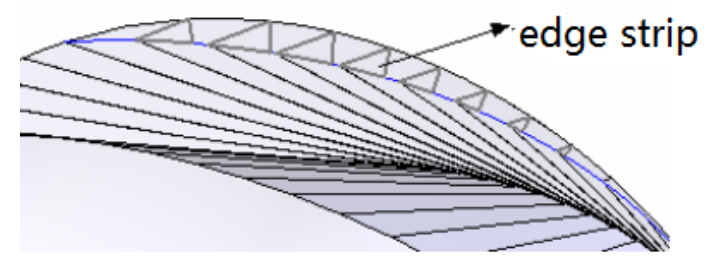

图 9 前刀面的刃带

\section{6 结论}

本文以球头立铣刀和砂轮实体的参数化建模为例, 简要介绍了利用 VB 对 SolidWorks 进行二次开发的 一般方法及步骤, 此方法避开了开发过程中的难点, 减少了工作量, 达到了开发目的。用此方法建立的球 头立铣刀加工仿真系统, 运行可靠, 大量节省了设计人员的时间和精力, 验证了前期所建数学模型的正确 性, 具有一定的实际意义。同时, 对 $\mathrm{CAD} / \mathrm{CAM}$ 软件的应用和推广也有重要的推动作用。

\section{参考文献:}

[1] Huang Zheng-sheng. Study on Grinding Parameters of Ball-nose End Mill about Analysis of Stress Field and High-speed Milling Modal[D]. Guizhou University, 2009.

[2] Lan Hua ,Jiang Daoshun. 3D Modeling Technology of Ball End Mill Based on SolidWorks[J]. Mechanical Engineer.2016.11,210-211.

[3] Hei Daquan. A Study on Parametric design and geometry parameters Optimization of Ball-end cutter[D]. Hunan University,2012.

[4] Ying Lv, Yao Bin. Research on Grinding Forming Cutter Location of Ball-nose End Mill with“S”Shape Major Cutting Edges [J]. Tool Technology.Vol.49,No10,2015,24-27.

[5] Zeng Linlin. Research on the Structure Design and Milling Performance of Indexable Ball-End Mill with Double-edged [D]. Xihua University, 2015.

[6] He Lin, Li Shuai, et al. Edge sharpening of the ball-end mill rakeface[J]. Machinery Design and Manufacture, 2009 (2): 
$250 \sim 251$.

[7] 江洪, 李仲兴, 邢启恩. SoildWorks2013 二次开发基础与实例教程[M]. 北京: 电子工业出版社, 2013

[8] 清源计算机工作室编著. Visual Basic6.0 开发宝典[M]. 北京: 机械工业出版社,2015

[9] Solidworks Corporation.Solidworks 2015 API help [Z]. 2015.

[10] Hei Daquan,Zhou Zhixiong. Optimization of geometric parameters of baii-end cutter based on the multiple nonlinear regression [J].Modern Manufacturing Engineering.2013.1,51-54.

[11] Jiang Hong, Wei Zheng, Wang Taowei, et al. SolidWorks Secondary Development Case Analysis [M]. Beijing: China Machine Press, 2014.

\section{References}

[1] Huang Zheng-sheng. Study on Grinding Parameters of Ball-nose End Mill about Analysis of Stress Field and High-speed Milling Modal [D]. Guizhou University, 2009.

[2] Lan Hua, Jiang Daoshun. 3D Modeling Technology of Ball End Mill Based on SolidWorks [J]. Mechanical Engineer.2016.11, 210-211.

[3] Hei Daquan. A Study on Parametric design and geometry parameters Optimization of Ball-end cutter [D]. Hunan University, 2012.

[4] Ying Lv, Yao Bin. Research on Grinding Forming Cutter Location of Ball-nose End Mill with "S" Shape Major Cutting Edges [J]. Tool Technology.Vol.49, No10, 2015, 24-27.

[5] Zeng Linlin. Research on the Structure Design and Milling Performance of Indexable Ball-End Mill with Double-edged [D]. Xihua University, 2015.

[6] He Lin, Li Shuai, et al. Edge sharpening of the ball-end mill rakeface [J]. Machinery Design and Manufacture, 2009 (2): 250 251.

[7] Jiang Hong, Li Zhongxing, Xing Qi'en. 2nd Development Foundation of SoildWorks2013 and Tutorial Examples [M].Beijing: Publishing House of Electronics Industry, 2013.

[8] Qingyuan Computer Studio Editor.VisualBasic6.0 Development Bownden [M].Beijing: Publishing House of Electronics Industry,2015

[9] Solidworks Corporation. Solidworks 2015 API help [Z]. 2015.

[10] Hei Daquan, Zhou Zhixiong. Optimization of geometric parameters of baii-end cutter based on the multiple nonlinear regression [J].Modern Manufacturing Engineering.2013.1, 51-54.

[11]Jiang Hong, Wei Zheng, Wang Taowei, et al. SolidWorks Secondary Development Case Analysis [M]. Beijing: China Machine Press, 2014. 